\title{
Ventilación mecánica en trauma de tórax severo: aspectos fisiopatológicos y nuevas perspectivas de manejo
}

\section{Mechanical ventilation in severe chest trauma: pathophysiological aspects and new management perspectives}

\author{
René R. Díaz ${ }^{(1)}$; Rubén Dueñas ${ }^{(2)}$; Ricardo Buitrago ${ }^{(3)}$; Gladys Canchila ${ }^{(4)}$; Marcela Poveda ${ }^{(5)}$
}

\section{RESUMEN}

El trauma severo de tórax es una patología con una alta morbilidad y mortalidad, siendo responsable aproximadamente de un $50 \%$ de las muertes en los pacientes politraumatizados. Los aspectos fisiopatológicos relacionados con el mecanismo de lesión, el estado de salud previo del paciente y el tiempo de evolución del trauma son factores importantes que determinarán el tipo de soporte ventilatorio que debe recibir el paciente que lo requiera. Los modos no convencionales de ventilación mecánica así como los dispositivos de asistencia ventilatoria se han convertido en herramientas útiles con un impacto importante en los resultados del paciente con trauma de tórax severo.

Palabras clave: trauma de tórax, contusión pulmonar, ventilación de alta frecuencia, dispositivos de asistencia pulmonar, ventilación con liberación de presiones de la vía aérea, ventilación mecánica diferencial.

\begin{abstract}
Severe chest trauma is a condition whose morbidity and mortality are high; it is accountable for roughly $50 \%$ of deaths of polytraumatized patients. The type of ventilatory support that is to be given to the patient who requires it depends in part on important factors such as the mechanism of injury, the patient's previous health condition, and the time of evolution of the trauma. Nonconventional ventilatory modalities, as well as lung-assist devices, have become useful tools with significant impact on the outcome of the patient with severe chest trauma.
\end{abstract}

Key words: chest trauma, pulmonary contusion, high-frequency ventilation, lung-assist devices, airway pressure release ventilation, differential mechanical ventilation

Rev Colomb Neumol 2011; 23(1): 22-25.

La ventilación mecánica del paciente con trauma de tórax es un reto para el intensivista debido a los cambios fisiológicos que ocurren a nivel de la ventilación, el intercambio gaseoso y flujo sanguíneo pulmonar como consecuencia del mecanismo de la lesión primaria y secundaria.

Los cambios que se desencadenan como consecuencia de la lesión secundaria son las alteraciones dadas por los mediadores de la respuesta inflamatoria sistémica (citocinas proinflamatorias, factor de necrosis tumoral, óxido nítrico, entre otras), que ocasionan una alteración en la membrana alvéolo capilar, aumentando su permeabilidad, reduciendo la capacidad de difusión de oxígeno y gases tanto en el pulmón lesionado como en el no afectado, lo cual ha sido demostrado en estudios experimentales en muestras de lavado broncoalveolar en animales (1). La severidad de la lesión primaria y secundaria se verá reflejada clínicamente como una lesión pulmonar aguda o SDRA (síndrome de dificultad respiratoria del adulto).

(1) Dr. Residente de tercer año Medicina Crítica y Cuidado Intesivo. Fundación Clínica Shaio. Universidad de la Sabana. Bogotá, Colombia.

(2) Dr, Neumólogo. Fundación Clínica Shaio. Universidad de la Sabana. Bogotá, Colombia.

(3) Dr. Profesor Medicina Crítica Fundación Clínica Shaio. Universidad de la Sabana. Bogotá, Colombia.

(4) Fisioterapeuta Fundación Clínica Shaio. Universidad de la Sabana. Bogotá, Colombia.

(5) Dra, Profesora Medicina Crítica Fundación Clínica Shaio. Universidad de la Sabana. Bogotá, Colombia.

Correspondencia: Doctor René R. Díaz renerdr10@yahoo.com

Recibido: 15 de febrero de 2011. Aceptado: 22 de febrero de 2011. 
Las principales alteraciones fisiológicas que se presentan durante un trauma de tórax severo son (1):

1. Disbalance de la relación ventilación-perfusión $(\mathrm{V} / \mathrm{Q})$.

2. Aumento del Shunt intrapulmonar .

3. Disminución heterogénea de la distensibilidad.

4. Aumento de la resistencia vascular pulmonar.

5. Reducción de la eliminación de CO2.

6. Disminución de la oxigenación.

\section{LESIÓN PRIMARIA}

Son tres los mecanismos de lesión primaria que se asocian al trauma de tórax (2):

1. Contusión pulmonar: se caracteriza por lesiones internas a nivel del parénquima pulmonar y/o vías aéreas pequeñas, siendo la magnitud de la lesión determinada por la interacción entre las fuerzas de cizallamiento y compresión directa sobre el tórax, así como por el área o la superficie del tórax sobre la cual el paciente ha recibido el impacto. La contusión pulmonar puede ser única ó múltiple teniendo como hallazgos clínicos hemotórax masivo, neumotórax a tensión o colapso lobar entre otros (2); a nivel microscópico se observa ocupación del espacio alveolar por secreciones, sangre y detritus resultado de la disrupción de la membrana alvéolo capilar lo cual alterará de acuerdo con la ley de Fick el intercambio gaseoso en el pulmón lesionado al aumentar el espesor de la membrana alveolo capilar.Cuando la contusión pulmonar es severa su mortalidad según las series publicadas alcanza hasta un $60 \%$.

2. Trauma penetrante: la magnitud de la lesión estará determinada por la profundidad y la fuerza aplicada sobre el tórax del paciente pudiendo afectar más de un órgano intratorácico (pleura, parénquima pulmonar, corazón, esófago, grandes vasos) asociándose principalmente con signos clínicos de deterioro ventilatorio y hemodinámico agudos que corresponderán a la presencia de hemotórax, neumotórax, taponamiento cardiaco o disección por ruptura vascular. La sobrevida de estos pacientes es mayor que los que sufren contusiones severas, dado que el reconocimiento de estas lesiones es más rápido y son llevados de manera temprana a salas de cirugía para su estabilización.

3. Lesión por onda explosiva: la magnitud de la lesión de estos pacientes es determinada por la intensidad y la distancia a la cual se encontraba el paciente de la primera y la segunda onda explosiva, lo cual puede desencadenar como lesiones intratorácicas, neumotórax a tensión, lesión de vía aérea o de tejido cardiaco e incluso contusión pulmonar $(1,2)$.
Como se mencionó previamente los objetivos primordiales del manejo del paciente con trauma de tórax severo radica en prevenir la hipoxia e hipoventilación y de presentarse debe ser manejada de manera adecuada para evitar mayores riesgos al paciente y futuras complicaciones. Si se realiza una atención ventilatoria temprana se reduce el tiempo de asistencia ventilatoria y por ende la presencia de infecciones asociadas (neumonía de inicio temprano), sin embargo, cuando ésta dura un tiempo mayor a cinco días aumenta el riesgo de desarrollar neumonía de inicio tardío (asociada al ventilador).

Actualmente, el trauma severo de tórax es la principal causa de mortalidad en pacientes politraumatizados alcanzando hasta $25 \%$ (2). La mayoría de modos ventilatorios carecen de evidencia clínica para hacer una recomendación del uso de alguno de ellos. Han sido empleados como estrategia de rescate en aquellos pacientes que persisten con lesión pulmonar asimétrica manejados de manera convencional, en quienes el uso del PEEP pueden desencadenar una hiperinsuflación del pulmón sano con el consecuente riesgo de barotrauma,o redistribución del flujo con el consiguiente aumento del shunt y agravar la hipoxemia.

\section{VENTILACIÓN MECÁNICA NO INVASIVA}

El uso de ventilación mecánica no invasiva con presión positiva continua en la vía aérea y su aplicación en trauma de tórax, actualmente, no cuenta con una evidencia clínica fuerte que permita hacer una recomendación en el manejo de esta patología. Sin embargo, existen algunas publicaciones que demuestran su utilidad en aquellos pacientes que cursan con contusiones pulmonares leves a moderadas con hipoxemia concomitante (menor índice de intubación orotraqueal y estancia hospitalaria) (2), siempre y cuando no existan contraindicaciones para el uso de este modo ventilatorio como deterioro del estado neurológico, obesidad mórbida, mal manejo de secreciones de la vía aérea, riesgo de broncoaspiración o trauma facial (3).

\section{VENTILACIÓN MECÁNICA DE ALTA FRE- CUENCIA}

Su uso cobra importancia en el manejo de lesiones asociadas al barotrauma secundarias a lesión pulmonar por onda explosiva y en contusiones pulmonares asimétricas donde la aplicación de estrategias convencionales de ventilación mecánica sobrellevan un mayor riesgo de presiones altas en la vía aérea (barotrauma y atelectrauma); además, en estudios recientes, su uso ha demostrado una mejoría en el intercambio gaseoso al generar un balance entre los mecanismos de convección y difusión en la entrega de oxígeno $(2,3)$. La ventilación de alta frecuencia también ha sido descrita en el trata- 
miento de fístulas bronco pleurales y traqueo bronquiales dado que la entrega del volumen de aire inspirado se hace sobre los límites del volumen residual favoreciendo de este modo el cierre del tracto fistuloso.

\section{VENTILACIÓN MECÁNICA CON LIBERA- CIÓN DE PRESIONES DE LA VIIA AÉREA (APRV)}

Descrito como modo ventilatorio no convencional desde 1980, mantiene una presión media de la vía aérea mayor al permitir el uso de presión positiva elevada durante un tiempo relativamente prolongado produciendo de esta manera una recuperación de la oxigenación; a su vez, permite programar un tiempo corto de presiones bajas (menor a 1 segundo) lo que facilita la eliminación de $\mathrm{CO} 2$. Su ventaja radica en que durante ambas fases del ciclo se pueden producir respiraciones espontáneas lo cual evita el desacondicionamiento de los músculos respiratorios así como favorece el reclutamiento de las zonas dependientes previamente atelectásicas (4).

\section{NAVA (NEURALLY ADJUST VENTILATORY ASSIST)}

Su utilidad radica en evitar la fatigabilidad temprana de los músculos respiratorios, mediante la detección a través de sensores que miden la actividad eléctrica del diafragma y de esta manera permite aportar una proporción del trabajo respiratorio del paciente de acuerdo al objetivo terapéutico que se plantea. Este modo ventilatorio mejora la sincronía paciente-ventilador, de igual manera, reduce las presiones inspiratorias y permite un destete ventilatorio bajo condiciones de capacidad vital críticas (5).

\section{VENTILACIÓN MECÁNICA DIFERENCIAL}

Con el advenimiento de los tubos endotraqueales de doble luz (diseñados por Carlens y Robertshaw), sobrevino la ventilación mecánica diferencial o ventilación de pulmón individual como modo ventilatorio no convencional para el manejo de patologías severas con comportamiento fisiopatológico heterogéneo como es el caso del pulmón con contusión severa, el cual se caracteriza por tener una distensibilidad reducida con respecto al menos lesionado. Se puede ventilar de manera diferencial uno solo o ambos pulmones, en este último caso empleando dos ventiladores bajo parámetros preestablecidos diferentes, iniciando con VT (volumen corriente) de 4 a $5 \mathrm{ml} / \mathrm{kg}$ el cual se titula de acuerdo a la presión meseta de cada pulmón seguido de aplicación de PEEP selectivo como medida de reclutamiento alveolar del pulmón mas lesionado sin provocar la hiperinsuflación del pulmón sano. El destete de la venti- lación diferencial se logra cuando la diferencia de VT y distensibilidad estática sea menor a 100 ml y $20 \%$ respectivamente (1).

La ventilación mecánica diferencial también puede ser aplicada en casos de presencia de fístula broncopleural (pos quirúrgica o infecciosa) cuando su manejo es difícil empleando las estrategias de manejo convencional, con el objetivo de brindar volumen corriente, frecuencia respiratoria y el menor PEEP posible al pulmón lesionado (1)

\section{DISPOSITIVOS DE ASISTENCIA EXTRACORPÓREA}

Debido a que las lesiones traumáticas del tórax se asocian a hemorragia alveolar, disminución del surfactante pulmonar y aumento de la tensión superficial, se reduce así la superficie de intercambio gaseoso; cambios que se asocian a un aumento del shunt fisiológico, hipoventilación alveolar e hipercapnia severa en estos pacientes. La experiencia con los dispositivos de asistencia pulmonar (interventional lung assist, iLA) viene en aumento tras ser usados con resultados alentadores en pacientes con insuficiencia respiratoria reversible con trauma severo de tórax, en pacientes heridos en combate durante las guerras de Irak y recientemente en Afganistan (5). El empleo del iLA se asocia a un incremento moderado de la oxigenación, gracias a que alcanza una tasa de extracción de CO2 aproximada de 11,5 litros por minuto. Para su uso se requiere de: 1.) una fuente externa de oxígeno (con flujos de 10-12 litros/ minuto), 2.) un sistema pasivo de shunt dado por una conexión femoro femoral, arterio-venosa (Figura 1) y 3.) una diferencia de presión entre la arteria y vena femorales de $60-80 \mathrm{mmHg}$ mandatoria. La membrana del dispositivo logra una superficie efectiva de intercambio de 1,3 $\mathrm{mt2}$. No requiere de fuentes externas de energía por cuanto es perfundido por el corazón, ni de un intercambiador de calor, dado que la pérdida del mismo por convección es despreciable. La contraindicación para su uso está dada por inestabilidad hemodinámica de origen cardiaco (choque cardiogénico) y el riesgo de isquemia de miembros inferiores. El uso de estos dispositivos va de la mano con la aplicación de ventilación mecánica protectora e incluso según reporte de casos publicados, se ha empleado durante el proceso de destete ventilatorio de modos no convencionales (NAVA) con resultados favorables (5).

\section{VENTILACIÓN MECÁNICA EN TRAUMA SEVERO DE TÓRAX}

Hasta el momento no hay evidencia clínica que demuestre un impacto en morbi mortalidad de los pacientes que cursan con lesión pulmonar bilateral pos 


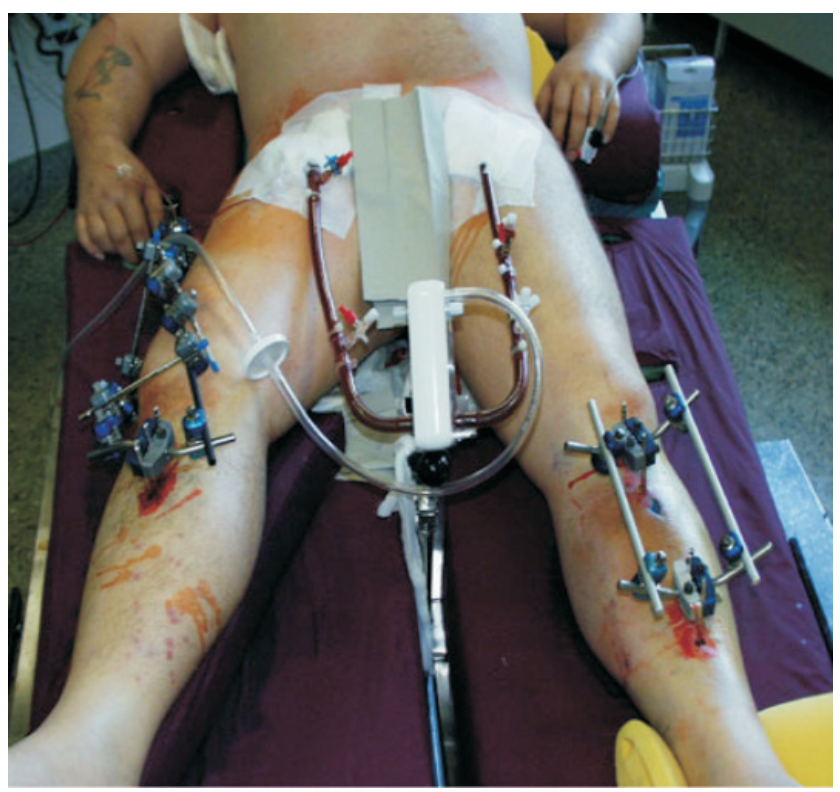

Figura 1. Paciente con trauma de tórax severo y trauma abdominal con insuficiencia respiratoria severa e hipercapnia severa con asistencia circulatoria extracorpórea con el dispositivo iLA.

traumática con el uso de los modos ventilatorios disponibles (convencionales, no convencionales). En reporte de casos se ha demostrado algún grado de beneficio en el SDRA pos contusión pulmonar con el uso de ventilación independiente con tubos de doble luz, bajo el concepto de que en esta condición patológica ambos pulmones muestran comportamientos diferentes en cuanto a distensibilidad y deterioro de la oxigenación, lo cual explicaría que aplicar un nivel más alto de PEEP al lado más afectado reclutaría las zonas dependientes que están mejor perfundidas y mal ventiladas para así lograr una mayor recuperación del intercambio de oxígeno (6). De manera contraria, existen reportes de casos del uso de la estrategia de ventilación diferencial junto con la adminsitración de óxido nítrico en forma de gas con el objetivo de producir una vasodilatación de las zonas no dependientes o mejor ventiladas y mejorar la relación V/ $\mathrm{Q}$, con resultados optimistas a corto plazo (6).

\section{CONCLUSIONES}

El manejo del paciente con trauma severo de tórax sigue siendo un reto para el médico intensivista, que no debe olvidar como objetivos primarios de su intervención terapéutica el limitar oportunamente la extensión de la lesión primaria y tratar de prevenir la presencia de la cascada inflamatoria o lesión secundaria al trauma de tórax inicial, sin embargo, en el momento no hay en la literatura evidencia de terapias médicas complementarias para la modulación de la reacción inflamatoria asociada a esta entidad.

Como se revisó previamente son varias las estrategias disponibles para el abordaje de este tipo de pacientes desde el punto de vista ventilatorio debiendo tener en cuenta siempre los probables cambios fisiopatológicos que en su gran mayoría son heterogéneos entre ambos pulmones de acuerdo al mecanismo y la severidad de la lesión torácica ocurrida, sin embargo, dichas opciones de intervención no cuentan hasta el momento con una evidencia clínica fuerte y deben realizarse estudios mayores para dar un mejor soporte epidemiológico a estas innovaciones terapéuticas.

\section{REFERENCIAS}

1. Sutyac JP. Pulmonary Contusions and Critical Care Management in Thoracic Trauma. Thoracic Surgery Clinics. 2007; 17: 11-23.

2. Kiraly L. Management of the crushed chest. CriticalCare Medicine 2010; 38 (9): S469-477.

3. Rico F. Mechanical ventilation strategies in massive chest trauma. Critical Care Clinics 2007; 23: 299-315.

4. Bonatti H. Trauma. Emergencies. Clinical of North America 2008; 26: 626-48.

5. Successful treatment of a severely injuried from Afghanistan with pumpless extracorporeal lung assist and neutrally adjusted ventilator support. International Journal Emergencies Medicine 2010; 3(3): 177-9. [http://www.ncbi.nlm.nih.gov.murzim. unisabana.edu.co/pubmed/21031042]

6. De Palma R. Blast Injuries. New England Journal of Medicine. 2005; 352: 1335-42. 